\title{
On maximum area polygons in a planar point set
}

\author{
Yatao Du and Ren Ding \\ Yatao Du received her M.Sc. and Ph.D. degrees from Hebei Normal University in \\ China. At present, she is teaching at Shijiazhuang Mechanical Engineering College in \\ China. Her research interests focus primarily on discrete and combinatorial geometry. \\ Ren Ding is a professor of mathematics and head of a Ph.D. program at Hebei Normal \\ University in China. His research interests focus primarily on discrete and combina- \\ torial geometry.
}

Let $P$ be a finite planar point set with no three points collinear, i.e. in general position. A subset $Q \subset P$ is called a convex polygon in $P$ if $Q$ forms the vertex set of a convex polygon. A convex polygon $Q \subset P$ is called an empty convex polygon if there is no point of $P$ in the interior of the convex hull of $Q$. Denote the area of the convex hull of $Q \subset P$ by $S(Q)$. Let

$$
\begin{aligned}
f_{k}(P) & =: \max \left\{\frac{S(Q)}{S(P)}: Q \text { is an empty convex } k \text {-gon with vertices in } P\right\}, \\
f_{k}(n) & =: \min \left\{f_{k}(P):|P|=n, P \text { is in general position }\right\} .
\end{aligned}
$$

A finite set of points in the plane is called in convex position if it forms the set of vertices of a convex polygon. Let $P$ be a finite set of points in convex position in the plane. Then a polygon $Q$ with vertices in $P$ is always an empty polygon. Let

$$
f_{k}^{\text {conv }}(n)=: \min \left\{f_{k}(P):|P|=n, P \text { is in convex position }\right\} .
$$

Es sei $P$ die Eckenmenge eines konvexen $n$-Ecks in der Ebene, und es sei $S(P)$ dessen Flächeninhalt. Wird eine $k$-elementige Teilmenge $Q \subset P$ dieser Ecken ausgewählt, so überdeckt das zugehörige $k$-Eck den Bruchteil $S(Q) / S(P)$ der Gesamtfläche. Man wird versuchen, durch geeignete Wahl von $Q$ diesen Flächenanteil m öglichst gross zu machen. In dem nachfolgenden Beitrag behandeln die Autoren das folgende MinimaxProblem: Welcher Bruchteil $S(Q) / S(P) 1$ ässt sich, unabhängig von der Form des Ausgangspolygons, durch geeignete Wahl von $Q$ garantiert erreichen? Beispielsweise finden die Autoren im Fall $|P|=5$ und $|Q|=4$, dass das Viereck bei richtiger Wahl der weggelassenen Ecke mindestens den Bruchteil $2 /(5-\sqrt{5})$ des Fünfecks überdeckt. Eine allgemeine Lösung des hier behandelten Problems wäre wünschbar. 
In [2] the authors studied $f_{3}^{\text {conv }}(n)$. In this paper we evaluate $f_{4}^{\text {conv }}(n)$, and more generally, $f_{n-1}^{\text {conv }}(n)$.

Theorem 1. $f_{4}^{\text {conv }}(5)=\frac{2}{5-\sqrt{5}}$.

Proof. Let $P$ be a convex 5-gon with vertices $A, B, C, D, E$ in clockwise order. Suppose that the 4-gon $A B C D$ is a maximum area quadrilateral in $P$. Given two triangles, there exists a unique affine transformation which transforms one triangle into another. So, without loss of generality, we may assume that $A=(0,0), B=(0,1), D=(1,0)$, $C=(a, b)(a>0, b>0)$. We always assume that $b \geq 1$, see Fig. 2. Indeed, when $b<1$, the distance from $B$ to the straight line $A D$ is greater than the distance from $C$ to the straight line $A D$, then we can reflect $P$ about a vertical line without changing the ratio of the areas. See Fig. 1. Relabel the vertices of $P$ to ensure that the distance from $C^{\prime}$ to the straight line $A^{\prime} D^{\prime}$ is greater than distance from $B^{\prime}$ to the straight line $A^{\prime} D^{\prime}$, and in this way we come to the case of $b \geq 1$.
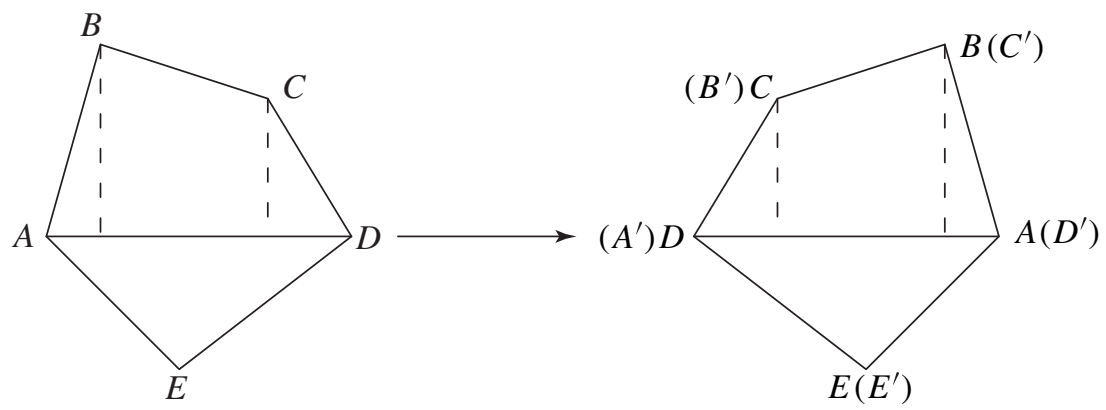

Fig. 1 The assumption $b \geq 1$

Let $Q_{1}, Q_{2}$ and $Q_{3}$ denote the 4-gons $A B C D, A B D E$ and $A C D E$, respectively, see Fig. 2. Let $f$ be the line through $A$ and $C$, and $f^{\prime}$ be the parallel line through $D$. Similarly, let $g$ be the line through $B$ and $D$, and $g^{\prime}$ be the parallel line through $A$. Since $Q_{1}=$ $A B C D$ is a maximum area quadrilateral in $P$, so $E$ lies completely above $f^{\prime}$ and $g^{\prime}$. Let $F=f^{\prime} \cap g^{\prime}$, then $F=\left(\frac{b}{a+b}, \frac{-b}{a+b}\right)$ and $E \in \triangle A D F$, and hence $P$ is always contained in the convex 5-gon $P^{\prime}=A B C D F$. Since $b \geq 1$, we have $S\left(Q_{3}\right) \geq S\left(Q_{2}\right)$; and since $S\left(Q_{1}\right) \geq S\left(Q_{3}\right)$, we have $S(\triangle A B C) \geq S(\triangle A D E)$. Suppose $E=\left(x_{0}, y_{0}\right)$,

$$
S(\triangle A B C)=\frac{a}{2}, S(\triangle A D E)=\frac{-y_{0}}{2} \Longrightarrow \frac{a}{2} \geq \frac{-y_{0}}{2} \Longrightarrow y_{0} \geq-a .
$$

So $E$ lies above the horizontal line $h: y=-a$. See Fig. 2, where $E$ does not appear since its position is depending. The figure shows only the case where $F$ lies below the line $h$.

Case 1. Suppose $F$ lies above the line $h$, then $\frac{-b}{a+b} \geq-a$, that is $\frac{b}{a+b} \leq a$. Notice that $P \subset P^{\prime}$ and so $S(P) \leq S\left(P^{\prime}\right)$.

$$
S\left(Q_{1}\right)=\frac{1}{2}(a+b), \quad S\left(P^{\prime}\right)=\frac{1}{2}(a+b)+\frac{b}{2(a+b)} .
$$




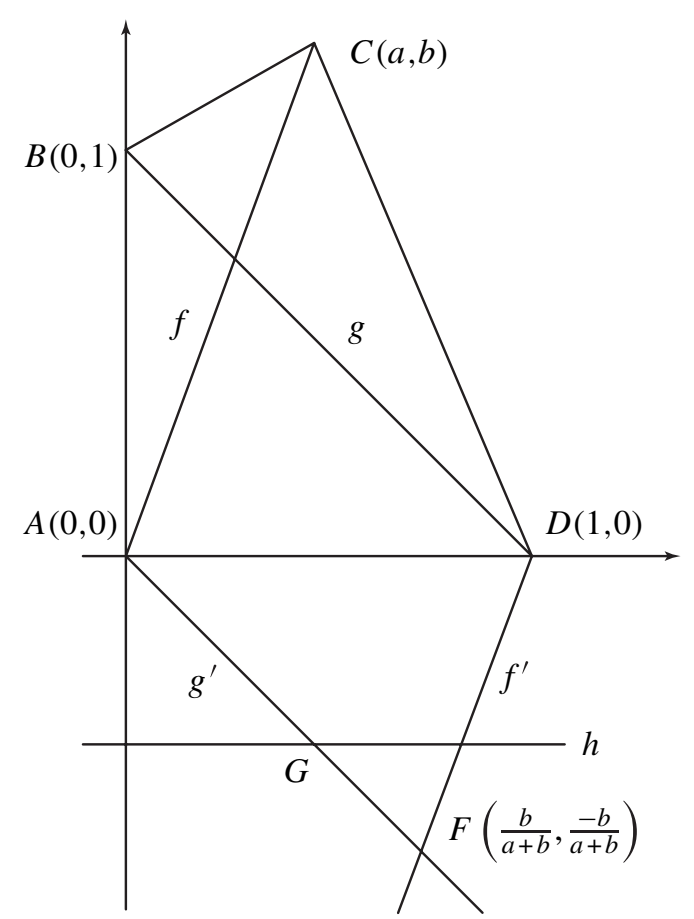

Fig. $2 E \in \triangle A D F, Q_{1}=A B C D, Q_{2}=A B D E, Q_{3}=A C D E$,

$$
P^{\prime}=A B C D F, P^{\prime \prime}=A B C D G, h: y=-a
$$

Subcase 1.1. Suppose $\frac{b}{a+b} \leq \frac{a}{b}$, then $\frac{a}{b} \geq \frac{\sqrt{5}-1}{2}$. By (*) and $b \geq 1$ we have

$$
\begin{gathered}
\frac{S(P)}{S\left(Q_{1}\right)} \leq \frac{S\left(P^{\prime}\right)}{S\left(Q_{1}\right)}=1+\frac{b}{(a+b)^{2}} \leq 1+\frac{b^{2}}{(a+b)^{2}}=1+\frac{1}{\left(\frac{a}{b}+1\right)^{2}} \leq \frac{5-\sqrt{5}}{2} \\
\Longrightarrow \frac{S\left(Q_{1}\right)}{S(P)} \geq \frac{2}{5-\sqrt{5}} .
\end{gathered}
$$

Subcase 1.2. Suppose $\frac{b}{a+b}>\frac{a}{b}$, then

$$
\frac{b}{a}>\frac{2}{\sqrt{5}-1} \Longrightarrow \frac{a}{a+b}<\frac{3-\sqrt{5}}{2}
$$

Recall that $\frac{b}{a+b} \leq a$, we have

$$
\begin{aligned}
\frac{S(P)}{S\left(Q_{1}\right)} \leq \frac{S\left(P^{\prime}\right)}{S\left(Q_{1}\right)} & \leq 1+\frac{b}{(a+b)^{2}} \leq 1+\frac{a}{a+b} \leq \frac{5-\sqrt{5}}{2} \\
& \Longrightarrow \frac{S\left(Q_{1}\right)}{S(P)} \geq \frac{2}{5-\sqrt{5}} .
\end{aligned}
$$


Case 2. Suppose $F$ lies below the line $h$ (see Fig. 2), then $\frac{-b}{a+b}<-a$, that is $\frac{b}{a+b}>a$, and since $E$ is above the horizontal line $h$, so $S(P) \leq S\left(P^{\prime \prime}\right)$, where $P^{\prime \prime}=A B C D G$ is a 5-gon with $G=g^{\prime} \cap h$. Since $g^{\prime}: y=-x, h: y=-a$, so $G=(a,-a)$.

$$
\begin{gathered}
S\left(P^{\prime \prime}\right)=\frac{1}{2}(a+b)+\frac{1}{2} a . \\
\frac{b}{a+b}>a \Longrightarrow \frac{b}{a+b}>\frac{a}{b} \Longrightarrow \frac{b}{a}>\frac{2}{\sqrt{5}-1} \Longrightarrow \frac{a}{a+b}<\frac{3-\sqrt{5}}{2} . \\
\frac{S(P)}{S\left(Q_{1}\right)} \leq \frac{S\left(P^{\prime \prime}\right)}{S\left(Q_{1}\right)}=1+\frac{a}{a+b} \leq \frac{5-\sqrt{5}}{2} \Longrightarrow \frac{S\left(Q_{1}\right)}{S(P)} \geq \frac{2}{5-\sqrt{5}} .
\end{gathered}
$$

From the above argument, we obtain that for any 5-point set $P$ in convex position we have $f_{4}(P) \geq \frac{2}{5-\sqrt{5}}$ and hence $f_{4}^{\text {conv }}(5) \geq \frac{2}{5-\sqrt{5}}$.

Let $a=\frac{\sqrt{5}-1}{2}, b=1$, and hence the line $h$ passes through $F$. Let $E=F$, then $\frac{S\left(Q_{1}\right)}{S(P)}=$ $\frac{a+b}{a+b+\frac{b}{a+b}}=\frac{2}{5-\sqrt{5}}$ by $(*)$, so $f_{4}^{\text {conv }}(5) \leq \frac{2}{5-\sqrt{5}}$.

Hence $f_{4}^{\text {conv }}(5)=\frac{2}{5-\sqrt{5}}$.

Theorem 2. $f_{4}^{\text {conv }}(6) \geq \frac{1}{4-\sqrt{5}}$.

Proof. Let $P$ be a convex 6-gon with vertices $A_{1}, A_{2}, A_{3}, A_{4}, A_{5}, A_{6}$ in clockwise order. Suppose that the 4-gon $Q$ is a maximum area quadrilateral in $P$, then $Q$ must be in one of the forms of $A_{i} A_{i+1} A_{i+2} A_{i+4}, A_{i} A_{i+1} A_{i+3} A_{i+4}$ or $A_{i} A_{i+1} A_{i+2} A_{i+3}$, where the addition in the subscript is modulo 6 .

Case 1. Suppose $Q=A_{i} A_{i+1} A_{i+2} A_{i+4}$.

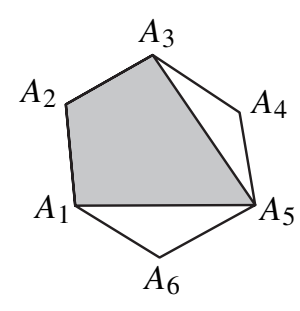

(a)

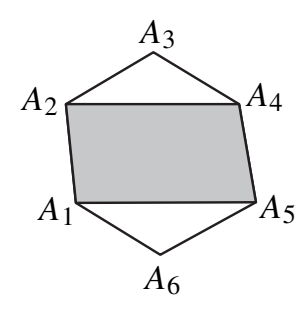

(b)

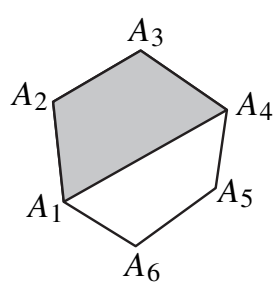

(c)

Fig. 3 Possible forms of maximum area quadrilaterals in $P$

Without loss of generality let $Q=A_{1} A_{2} A_{3} A_{5}$, as shown in Fig. 3(a). Let $P_{1}=$ $A_{1} A_{2} A_{3} A_{4} A_{5}, P_{2}=A_{1} A_{2} A_{3} A_{5} A_{6}$. Then $Q$ is also the maximum area quadrilateral in $P_{1}$ and in $P_{2}$. By Theorem 1 , we have

$$
\frac{S(P)}{S(Q)}=\frac{S\left(P_{1}\right)+S\left(P_{2}\right)-S(Q)}{S(Q)} \leq \frac{5-\sqrt{5}}{2}+\frac{5-\sqrt{5}}{2}-1=4-\sqrt{5} .
$$


Thus

$$
\frac{S(Q)}{S(P)} \geq \frac{1}{4-\sqrt{5}}
$$

Case 2. Suppose $Q=A_{i} A_{i+1} A_{i+3} A_{i+4}$, see Fig. 3(b). By the same argument as in Case 1 we obtain the required conclusion.

Case 3. Suppose $Q=A_{i} A_{i+1} A_{i+2} A_{i+3}$, that is, $Q$ is formed by four consecutive vertices of $P$. Without loss of generality let $Q=A_{1} A_{2} A_{3} A_{4}$, as shown in Fig. 3(c). By using an affine transformation we may assume that $A_{1}=(0,0), A_{2}=(0,1), A_{4}=(1,0)$, $A_{3}=(a, b)(a>0, b \geq 1)$. See Fig. 4 .

Let $f$ be the line through $A_{1}$ and $A_{3}$, and let $f^{\prime}$ be the parallel line through $A_{4}$. Similarly, let $g$ be the line through $A_{2}$ and $A_{4}$, and let $g^{\prime}$ be the parallel line through $A_{1}$. Let $F=$ $f^{\prime} \cap g^{\prime}$, so $F=\left(\frac{b}{a+b}, \frac{-b}{a+b}\right)$. Similar to the proof of Theorem 1, here $A_{5}, A_{6} \in \triangle A_{1} A_{4} F$ and $A_{5}, A_{6}$ lie above the horizontal line $h: y=-a$. So $P$ must be contained in the convex 5-gon $P^{\prime}=A_{1} A_{2} A_{3} A_{4} F$.

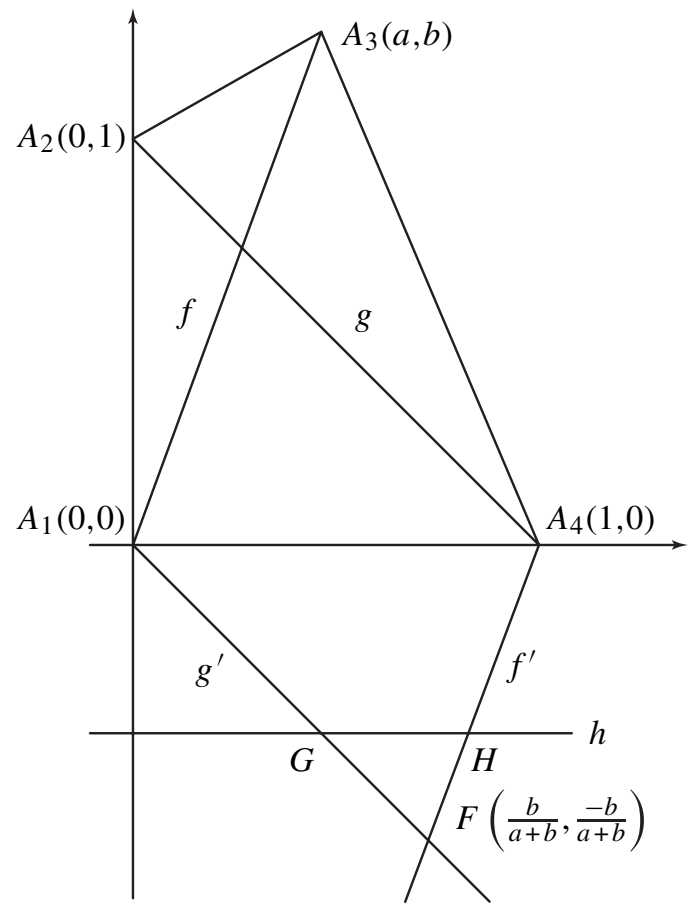

Fig. 4

Subcase 3.1 Suppose $F$ lies above the line $h$, then $\frac{-b}{a+b} \geq-a$, i.e. $\frac{b}{a+b} \leq a$, so $A_{5}$ and $A_{6}$ obviously lie above the line $h$. By the same argument as in Case 1 in proving Theorem 1 , we have

$$
\frac{S(P)}{S(Q)} \leq \frac{5-\sqrt{5}}{2}<4-\sqrt{5} \Longrightarrow \frac{S(Q)}{S(P)}>\frac{1}{4-\sqrt{5}} \text {. }
$$


Subcase 3.2 Suppose $F$ lies below the line $h$, then $\frac{-b}{a+b}<-a$, i.e. $\frac{b}{a+b}>a$, so $P$ must be contained in the hexagon $P^{\prime \prime}=A_{1} A_{2} A_{3} A_{4} H G$ with $G=g^{\prime} \cap h$ and $H=f^{\prime} \cap h$, where $G=(a,-a)$, and $H=\left(1-\frac{a^{2}}{b},-a\right)$. The area of $P^{\prime \prime}$ equals the area of the 4-gon $Q$ plus the area of the 4-gon $A_{1} A_{4} H G$, hence

$$
\begin{gathered}
S\left(P^{\prime \prime}\right)=\frac{1}{2}(a+b)+a-\frac{a^{3}+a^{2} b}{2 b}, \\
\frac{S(P)}{S(Q)} \leq \frac{S\left(P^{\prime \prime}\right)}{S(Q)}=1+\frac{2 a}{a+b}-\frac{a^{3}+a^{2} b}{(a+b) b}<1+\frac{2 a}{a+b}<4-\sqrt{5} \\
\Longrightarrow \frac{S(Q)}{S(P)}>\frac{1}{4-\sqrt{5}},
\end{gathered}
$$

and hence $f_{4}^{\text {conv }}(6) \geq \frac{1}{4-\sqrt{5}}$.

Lemma A. Let $P_{n}$ be the set of vertices of a regular $n$-gon, and let $r_{4}(n)=: f_{4}\left(P_{n}\right)$, then

$$
\begin{aligned}
& r_{4}(n)=\frac{4}{n \sin \frac{2 \pi}{n}} \quad \text { when } n \equiv 0 \quad(\bmod 4) \\
& r_{4}(n)=\frac{3 \cos \frac{\pi}{2 n}+\cos \frac{3 \pi}{2 n}}{n \sin \frac{2 \pi}{n}} \quad \text { when } n \equiv 1 \quad \text { or } 3 \quad(\bmod 4) \\
& r_{4}(n)=\frac{4 \cos \frac{\pi}{n}}{n \sin \frac{2 \pi}{n}} \quad \text { when } n \equiv 2 \quad(\bmod 4)
\end{aligned}
$$

Proof. Suppose that the maximum area quadrilateral $A B C D$ with vertices in $P_{n}$ divides

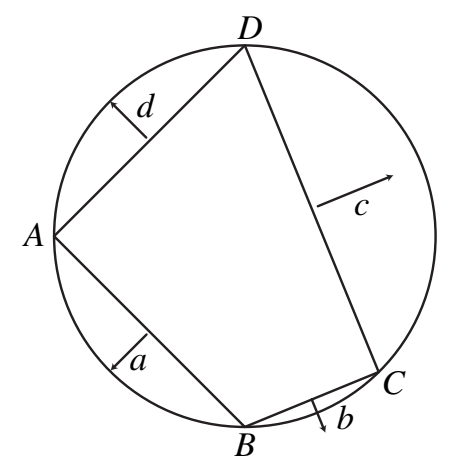

Fig. 54 chains on the boundary of convex hull of $P_{n}$

the boundary of the convex hull of $P_{n}$ into four chains $\widehat{A B}, \widehat{B C}, \widehat{C D}$ and $\widehat{D A}$ with $a, b, c$ and $d$ edges, respectively, as shown in Fig. 5. 
First, we prove that any two of these numbers differ at most by 1 .

Case 1. Suppose for two adjacent numbers, say, $b$ and $c$ we have $c-b \geq 2$. See Fig. $6(a)$.

Let $E$ be the nearest point of $P_{n}$ to $C$ in anticlockwise order. Observe that since $c-b \geq 2$, the numbers of points of $P_{n}$ on $\widehat{E D}$ is greater than that on $\widehat{B C} . S(\triangle D C E)>S(\triangle B C E)$, because both triangles have the same base $C E$, and the distance from $D$ to the straight line $C E$ is greater than the distance from $B$ to the straight line $C E$. Then the area of the 4-gon $A B D E$ is greater than the area of the 4-gon $A B C D$, contradicting the choice of the 4-gon $A B C D$.

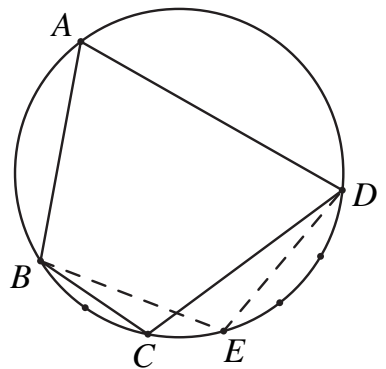

(a) $b=2, c=4$

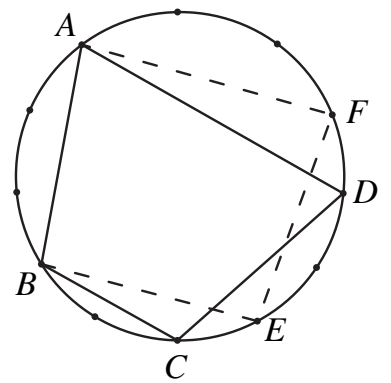

(b) $a=c=3, b=2, d=4$

Fig. 6

Case 2. Suppose for two nonadjacent numbers, say, $b$ and $d$ we have $d-b \geq 2$. From Case 1 , we need only to consider the cases $a=c=b+1$, and $d=b+2$, as shown in Fig. 6(b).

Let $E$ be the nearest point of $P_{n}$ to $C$ and $F$ be the nearest point of $P_{n}$ to $D$ in anticlockwise order. Then $S(A B C D)=S(A B E D)<S(A B E F)$, contradicting the choice of the 4-gon $A B C D$.

Therefore, we conclude that a maximal area quadrilateral in $P_{n}$ splits the boundary of the convex hull of $P_{n}$ into four chains whose numbers of edges are $\{t, t, t, t\},\{t, t, t, t+$ $1\},\{t, t, t+1, t+1\},\{t, t+1, t+1, t+1\}$, when $n \equiv 0,1,2,3(\bmod 4)$, respectively. An easy computation leads to the claimed formulas.

Notice that each $r_{4}(n)$ is a decreasing function. Thus we can deduce that

$$
\lim _{n \rightarrow \infty} r_{4}(n)=\frac{2}{\pi}
$$

Lemma B. Let $B$ be a compact convex region in the plane and $B_{k}$ be a largest area $k$-gon inscribed in $B$. Then area $\left(B_{k}\right) \geq \operatorname{area}(B) \frac{k}{2 \pi} \sin \frac{2 \pi}{k}$, where equality holds if and only if $B$ is an ellipse.

From Theorem 2, Lemma A and Lemma B, the following results can be easily obtained: 
Theorem 3. For planar point sets in convex position of size $n \geq 7$, we have

$$
\frac{2}{\pi} \leq f_{4}^{\text {conv }}(n) \leq r_{4}(n) \text {. }
$$

Theorem 4. $\frac{1}{4-\sqrt{5}} \leq f_{4}^{\text {conv }}(6) \leq r_{4}(6)=\frac{2}{3}$.

Theorem 5. $f_{n-1}^{\text {conv }}(n) \leq 1-\frac{2\left(1-\cos \frac{2 \pi}{n}\right)}{n} \quad(n \geq 4)$.

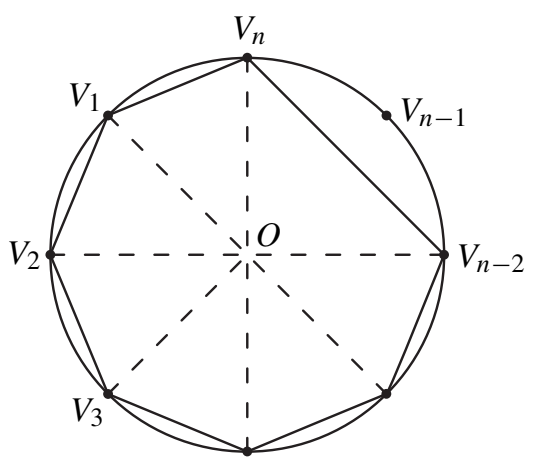

Fig. 7

Proof. Let $P_{n}=V_{1} V_{2} V_{3} \ldots V_{n}$ be a regular $n$-gon with circumradius equal to 1 and circumcenter at $O$, then every $(n-1)$-gon $Q$ in $P_{n}$ has the same area. See Fig. 7.

$$
\begin{gathered}
S\left(P_{n}\right)=n S\left(\triangle V_{1} O V_{2}\right)=\frac{n}{2} \sin \frac{2 \pi}{n}, \\
S(Q)=(n-2) S\left(\triangle V_{1} O V_{2}\right)+S\left(\triangle V_{n-2} O V_{n}\right)=\frac{n-2}{2} \sin \frac{2 \pi}{n}+\frac{1}{2} \sin \frac{4 \pi}{n} \\
\Longrightarrow \frac{S(Q)}{S\left(P_{n}\right)}=1-\frac{2\left(1-\cos \frac{2 \pi}{n}\right)}{n} .
\end{gathered}
$$

Hence $f_{n-1}^{\text {conv }}(n) \leq 1-\frac{2\left(1-\cos \frac{2 \pi}{n}\right)}{n}$ by the definition of $f_{n-1}^{\text {conv }}(n)$.

A few words on why the topic should be discussed might be necessary. The study of exact algorithms for robot motion planning forms a major subarea of computational geometry, with connections also to symbolic and algebraic computation. Motion planning is useful not only for computer control of actual robots but also for assembly planning and to computer animation. A related problem is found in robot motion planning where one might want to approximate the shape of a robot moving from one room to the next through a narrow door, the numerical bounds for the ratio of the maximum area quadrilateral to the robot body area could be a measure of how good an approximation is. For details see [1].

Acknowledgement. We are very grateful to Prof. Masatsugu Urabe for his great help in sending us his manuscript and some related papers. We are also very grateful to the anonymous referee for his comments and suggestions. 


\section{References}

[1] Fleischer, R.; Mehlhorn, V.; Rote, G.; Welzl, E.; Yap, C.: Simultaneous inner and outer approximation of shapes. Algorithmica 8 (1992), 365-389.

[2] Hosono, K.; Hurtado, F.; Urabe, M.; Urrutia, J.: On the triangle with the maximum area in a planar point set, Lecture Notes in Comput. Sci. 3330 (2005), 102-107.

Yatao Du

Department of Mathematics

Shijiazhuang Mechanical Engineering College

Shijiazhuang, 050003, China

e-mail: dyt77@eyou.com

Ren Ding*

Department of Mathematics

Hebei Normal University

Shijiazhuang, 050016, China

e-mail: rending@hebtu.edu.cn rending@heinfo.net

*This research was supported by National Natural Science Foundation of China (10571042), NSF A2005000144, 05CXJJ02 and L2004202. 